\title{
Hamiltonian embeddings from triangulations
}

\author{
Mike J. Grannell, Terry S. Griggs \\ Department of Mathematics \\ The Open University \\ Walton Hall \\ Milton Keynes MK7 6AA \\ U.K. \\ Jozef Šrán̆ \\ Department of Mathematics \\ Faculty of Civil Engineering \\ Slovak University of Technology \\ Radlinského 11 \\ 81368 Bratislava \\ SLOVAKIA
}

February 26, 2007

This is a preprint of an article accepted for publication in the Bulletin of the London Mathematical Society (C)2007 (copyright owner as specified in the journal). 


\begin{abstract}
A Hamiltonian embedding of $K_{n}$ is an embedding of $K_{n}$ in a surface, which may be orientable or non-orientable, in such a way that the boundary of each face is a Hamiltonian cycle. Ellingham and Stephens recently established the existence of such embeddings in non-orientable surfaces for $n=4$ and $n \geq 6$. Here we present an entirely new construction which produces Hamiltonian embeddings of $K_{n}$ from triangulations of $K_{n}$ when $n \equiv 0$ or $1(\bmod 3)$. We then use this construction to obtain exponential lower bounds for the numbers of nonisomorphic Hamiltonian embeddings of $K_{n}$.
\end{abstract}




\section{Running head:}

Hamiltonian embeddings

\section{Corresponding author:}

Mike Grannell, Department of Mathematics, The Open University, Walton Hall, Milton Keynes MK7 6AA, United Kingdom.

email: m.j.grannell@open.ac.uk

Other author email addresses:

Terry Griggs: t.s.griggs@open.ac.uk

Jozef Širáň: <jozef.siran@stuba.sk>

\section{AMS classification:}

$05 \mathrm{C} 10$

\section{Keywords:}

Topological embedding, complete graph, Hamiltonian decomposition. 


\section{Introduction}

A Hamiltonian embedding of $K_{n}$, the complete graph of order $n$, is an embedding of $K_{n}$ in a surface, which may be orientable or non-orientable, in such a way that the boundary of each face is a Hamiltonian cycle. The recent paper by Ellingham and Stephens [5] established the existence of such embeddings in non-orientable surfaces for $n=4$ and $n \geq 6$. In this paper we present an entirely new construction which, by surgery on a surface triangulation of $K_{n}$, generates a Hamiltonian embedding of $K_{n}$ on a surface of higher genus. This novel construction may be used to establish the existence of $2^{a n-o(n)}$ nonisomorphic Hamiltonian embeddings of $K_{n}$ for $n \equiv 0$ or $1(\bmod 3)$, where $a>0$ is a constant. For certain residue classes of $n$ this lower bound may be improved to $2^{a n^{2}-o\left(n^{2}\right)}$.

Ringel, Youngs and others established the existence of triangulations of $K_{n}$ for $n \equiv 0$ or $1(\bmod 3)$ in the course of proving the Heawood map colouring conjecture [11]. To elaborate briefly: if $n \equiv 0,3,4$ or $7(\bmod 12)$ then there is a triangulation of $K_{n}$ in an orientable surface, while if $n \equiv 0$ or $1(\bmod 3)$ and $n \neq 3,4$ or 7 , then there is a triangulation of $K_{n}$ in a non-orientable surface. In a triangulation, each face is as small as possible. At the opposite extreme, for every $n$ there exists an embedding of $K_{n}$ having a single face (see, for example, [9]). Around this single face every vertex appears $n-1$ times. The problem of constructing Hamiltonian embeddings of $K_{n}$ is intermediate between the two extremes - the face lengths are as large as possible subject to the restriction that no vertex is repeated on the boundary of any face.

In a Hamiltonian embedding of $K_{n}$, the number of faces is $n-1$. In the nonorientable case, Euler's formula gives the genus as $\gamma=(n-2)(n-3) / 2$. In the orientable case, the genus is $g=(n-2)(n-3) / 4$, which implies that $n \equiv 2$ or 3 $(\bmod 4)$ is a necessary condition for the embedding.

We assume that the reader is familiar with the basic terminology and methods of topological graph theory, such as may be found in [9, 11], in particular the representation of embeddings by rotation schemes.

\section{The construction}

As remarked above, for $n \equiv 0$ or $1(\bmod 3)$, there exists a triangulation of $K_{n}$ in a surface. Our construction starts with any such triangulation of $K_{n}$; whether the triangulation is in an orientable or non-orientable surface is immaterial. To avoid trivial cases we assume that $n \geq 4$.

\section{Construction 2.1}


Take a triangulation of $K_{n}$ on the vertex set $\left\{\infty, a_{1}, a_{2}, \ldots, a_{n-1}\right\}$ and, without loss of generality, take the rotation scheme to have the following form.

$$
\begin{array}{rllllllll}
\infty & : & a_{1} & a_{2} & a_{3} & a_{4} & \ldots & a_{n-2} & a_{n-1} \\
a_{1} & : & \infty & a_{2} & b_{1,1} & b_{1,2} & \ldots & b_{1, n-4} & a_{n-1} \\
a_{2} & : & \infty & a_{3} & b_{2,1} & b_{2,2} & \ldots & b_{2, n-4} & a_{1} \\
\vdots & & & & & & \vdots & & \vdots \\
a_{i} & : & \infty & a_{i+1} & b_{i, 1} & b_{i, 2} & \ldots & b_{i, n-4} & a_{i-1} \\
\vdots & & & & & & \vdots & & \vdots \\
a_{n-1} & : & \infty & a_{1} & b_{n-1,1} & b_{n-1,2} & \ldots & b_{n-1, n-4} & a_{n-2}
\end{array}
$$

where, for each $i=1,2, \ldots, n-1,\left(b_{i, 1} b_{i, 2} \ldots b_{i, n-4}\right)$ is some permutation of $\left\{a_{1}, a_{2}, \ldots, a_{n-1}\right\} \backslash\left\{a_{i-1}, a_{i}, a_{i+1}\right\}$, with subscript arithmetic modulo $n-1$.

From the $n$ lines of the rotation scheme, create $n-1$ Hamiltonian cycles by discarding the first line and, for each $i$, replacing the line corresponding to $a_{i}$ by the cycle $A_{i}=\left(\infty a_{i} a_{i+1} b_{i, 1} b_{i, 2} \ldots b_{i, n-4} a_{i-1}\right)$. It is easy to see that these cycles form a Hamiltonian decomposition of $2 K_{n}$. The Hamiltonian face corresponding to $A_{i}$ is formed from the triangular faces that comprise the rotation at $a_{i}$ in the original triangulation, with the triangle $\left(\infty a_{i} a_{i+1}\right)$ removed. It remains to show that these Hamiltonian faces may be sewn together along common edges to produce a Hamiltonian embedding of $K_{n}$. In order to prove this, it is only necessary to prove that the resulting rotation about any vertex comprises a single cycle of length $n-1$, rather than a set of shorter cycles with total length $n-1$. Note that a section of a cycle boundary $(\ldots a b c \ldots)$ gives rise to a part of the rotation about $b$ having the form $b: \ldots c c \ldots$ (of course, the direction of the rotation at $b$ is not determined).

Consider first the point $\infty$. The rotation about this point obtained from the Hamiltonian cycles is

$$
\infty \quad: \quad a_{1} \quad a_{2} \quad \ldots \quad a_{n-1}
$$

which is a single cycle of length $n-1$.

Consider next any of the remaining points, say $a_{i}$. In the original triangulation, the rotation

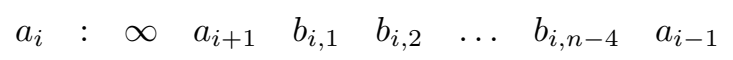

implies that, in this triangulation, the rotations about $a_{i+1}, b_{i, 1}, b_{i, 2}, \ldots, b_{i, n-5}$, $b_{i, n-4}, a_{i-1}$ contain the following sequences.

$$
\begin{array}{lllrrrl}
a_{i+1} & : & \ldots & b_{i, 1} & a_{i} & \infty & \ldots \\
b_{i, 1} & : & \ldots & b_{i, 2} & a_{i} & a_{i+1} & \ldots \\
b_{i, 2} & : & \ldots & b_{i, 3} & a_{i} & b_{i, 1} & \ldots \\
\vdots & & & & \vdots & & \\
b_{i, n-5} & : & \ldots & b_{i, n-4} & a_{i} & b_{i, n-6} & \ldots \\
b_{i, n-4} & : & \ldots & a_{i-1} & a_{i} & b_{i, n-5} & \ldots \\
a_{i-1} & : & \ldots & \infty & a_{i} & b_{i, n-4} & \ldots
\end{array}
$$


These sequences for $a_{i+1}, b_{i, 1}, b_{i, 2}, \ldots, b_{i, n-4}$ appear in the corresponding Hamiltonian cycles, while for $A_{i-1}$ and $A_{i}$ we have

$$
\begin{aligned}
& A_{i-1}=\left(\ldots a_{i-1} a_{i} b_{i-1,1} \ldots\right)=\left(\ldots a_{i-1} a_{i} b_{i, n-4} \ldots\right), \\
& A_{i}=\left(\ldots \infty a_{i} a_{i+1} \ldots\right) \text {. }
\end{aligned}
$$

These sequences enable us to construct the rotation about $a_{i}$ in the embedding of the Hamiltonian cycles. For $n$ even it is

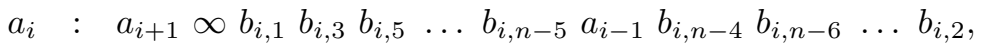

while for $n$ odd it is

$$
a_{i} \quad: \quad a_{i+1} \infty b_{i, 1} b_{i, 3} b_{i, 5} \ldots b_{i, n-4} a_{i-1} b_{i, n-5} b_{i, n-7} \ldots b_{i, 2} .
$$

In either case, this is a cycle of length $n-1$, and this completes the verification of the construction.

To consider the question of orientability, delete the point $\infty$ and the edges incident with $\infty$ from the embedding to obtain a single face embedding of $K_{n-1}$ with boundary

$$
\left(a_{1} a_{2} b_{1,1} b_{1,2} \ldots b_{1, n-4} a_{n-1} a_{1} b_{n-1,1} b_{n-1,2} \ldots b_{n-1, n-4} a_{n-2} a_{n-1} \ldots b_{2, n-4}\right) .
$$

If, in the order given, any subsequence of the form $a_{j} a_{j+1}$ appears twice in this boundary then the embedding of $K_{n-1}$, and hence that of $K_{n}$, must be nonorientable. When the original triangulation of $K_{n}$ is orientable this will happen for every $j=1,2, \ldots, n-1$. This is because each directed edge $a_{j} a_{j+1}$ must appear precisely once in one of the rotations $a_{i}: \infty a_{i+1} b_{i, 1} b_{i, 2} \ldots b_{i, n-4} a_{i-1}$. Thus an orientable triangulation of $K_{n}$ will, by this construction, produce a nonorientable Hamiltonian embedding of $K_{n}$. Although it appears conceivable that a non-orientable triangulation might produce an orientable Hamiltonian embedding of $K_{n}$ for $n \equiv 3,6,7$ or $10(\bmod 12)$, we have no examples of this and such situations seem likely to be rare.

\section{$3 \quad$ Exponential lower bounds}

In this section we establish lower bounds for the numbers of nonisomorphic Hamiltonian embeddings of $K_{n}$ for $n$ lying in certain residue classes. The supporting surface may be either orientable or nonorientable. We start with a lemma.

Lemma 3.1 A Hamiltonian embedding of $K_{n}, n \equiv 0$ or $1(\bmod 3)$, can be obtained from at most $2 n$ distinct triangulations of $K_{n}$ by means of Construction 2.1. 
Proof. Given a Hamiltonian embedding of $K_{n}$ on a fixed set of $n$ points, we check each point in turn to see if it can play the role of the point $\infty$ in the construction, and we show that, for each point, this can happen in at most two ways. So, take a point $h^{*}$ and suppose that the rotation at $h^{*}$ in the Hamiltonian embedding is

$$
h^{*}: h_{1} h_{2} \ldots h_{n-1}
$$

Then the Hamiltonian cycles may be taken as

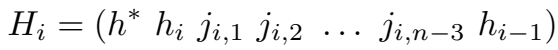

where $\left(j_{i, 1} j_{i, 2} \ldots j_{i, n-3}\right)$ is some permutation of $\left\{h_{1}, h_{2}, \ldots, h_{n-1}\right\} \backslash\left\{h_{i}, h_{i-1}\right\}$.

In order to be derived from the construction, we must either have $j_{i, 1}=h_{i+1}$ for every $i=1,2, \ldots, n-1$, or $j_{i, n-3}=h_{i-2}$ for every $i=1,2, \ldots, n-1$. In the former case, the rotations in the triangulation (assuming it exists) are determined as

$$
\begin{aligned}
& h^{*}: h_{1} h_{2} \ldots h_{n-1} \\
& h_{i}: h^{*} h_{i+1} j_{i, 2} j_{i, 3} \ldots j_{i, n-3} h_{i-1} \quad(i=1,2, \ldots, n-1) .
\end{aligned}
$$

In the latter case the rotations in the triangulation (assuming it exists) must be

$$
\begin{aligned}
& h^{*} \quad: \quad h_{1} h_{2} \ldots h_{n-1} \\
& h_{i}: h^{*} h_{i-1} j_{i+1, n-4} j_{i+1, n-5} \ldots j_{i+1,1} h_{i+1} \quad(i=1,2, \ldots, n-1) .
\end{aligned}
$$

The result now follows.

We now prove a result from which lower bounds may easily be deduced.

Theorem 3.1 If there exist $M$ nonisomorphic triangulations of $K_{n}, n \equiv 0$ or 1 (mod 3$)$, then there exist at least $M / 4 n^{2}(n-1)$ nonisomorphic Hamiltonian embeddings of $K_{n}$.

Proof. From $M$ nonisomorphic triangulations of $K_{n}$, it is possible to construct at least $M n ! / 2 n(n-1)$ distinct triangulations of $K_{n}$ on a common point set by applying all possible $n$ ! permutations of the points and noting that the largest possible order of an automorphism group of such a triangulation is $2 n(n-1)$. From each of these distinct triangulations we may construct a Hamiltonian embedding of $K_{n}$ using Construction 2.1. By Lemma 3.1, each such embedding can be obtained from at most $2 n$ distinct triangulations. Hence there are at least $M n ! / 4 n^{2}(n-1)$ distinct Hamiltonian embeddings on a common point set. The largest possible size of an isomorphism class for such an embedding is $n$ !. Hence there are at least $M / 4 n^{2}(n-1)$ nonisomorphic Hamiltonian embeddings of $K_{n}$.

Corollary 3.1.1 For $n \equiv 0$ or $1(\bmod 3)$ there are at least $2^{n / 6-o(n)}$ nonisomorphic Hamiltonian embeddings of $K_{n}$.

Proof. For $n \equiv 0$ or $1(\bmod 3)$, Korzhik and Voss [10] established that there are at least $2^{n / 6-o(n)}$ nonisomorphic triangulations of $K_{n}$. The result follows immediately from this and the Theorem. 
Corollary 3.1.2 For $n \equiv 1,7$ or $9(\bmod 18)$ there are at least $2^{n^{2} / 54-o\left(n^{2}\right)}$ nonisomorphic Hamiltonian embeddings of $K_{n}$.

Proof. Firstly we note that for each $n \equiv 3(\bmod 6)$ there is a face 2 -colourable triangulation of $K_{n}$ having a parallel class of faces (that is, a set of faces covering all $n$ vertices, each precisely once) in each colour class.

The orientable triangulations of $K_{n}, n \equiv 3(\bmod 12)$, given by Ringel [11] are face 2-colourable because the current graphs employed to construct these embeddings are bipartite. The Steiner triple systems involved in these embeddings are those produced by the Bose construction (see, for example, [3]) from the group $\left(\mathcal{Z}_{n / 3},+\right)$, and indeed a direct construction of the embeddings from these Steiner systems is given in [6]. The Bose construction produces Steiner triple systems having a parallel class, and so these orientable triangulations each contain a parallel class of faces in each colour class. Similarly, the nonorientable triangulations of $K_{n}, n \equiv 9(\bmod 12)$, also given by Ringel [11] are face 2-colourable since the cascades used to construct them are bipartite. As shown in [1], the Steiner triple systems involved here are also copies of Bose systems and hence the embeddings again have a parallel class of faces in each colour class. In fact, Ducrocq and Sterboul [4] also give a direct construction producing face 2-colourable triangulations of $K_{n}$ in nonorientable surfaces for all $n \equiv 3(\bmod 6), n \geq 9$, with the Steiner triple systems involved being copies of Bose systems.

Secondly we note that for $n \equiv 1(\bmod 6)$, Grannell and Korzhik [8] proved that there is a face 2-colourable triangulation of $K_{n}$ in a nonorientable surface. And we also remark that for all $n \equiv 7(\bmod 12)$, Youngs [12] gives a variety of embeddings, including face 2-colourable triangulations.

Having made these prelimnary observations, we can now use two recursive constructions to produce our lower bounds.

Applying the $n \rightarrow 3 n-2$ construction for triangular embeddings given in [2] establishes that for $n \equiv 1$ or $7(\bmod 18)$ there are at least $2^{n^{2} / 54-o\left(n^{2}\right)}$ nonisomorphic triangulations of $K_{n}$.

The paper [7] gives an $n \rightarrow m n$ construction for orientable triangulations but, as remarked in that paper, the method is easily extended to the nonorientable case. In the case $m=3$ it requires a face 2-colourable triangulation of $K_{9}$ and a parallel class of faces in one of the colour classes of the original $K_{n}$ triangulation. This construction then establishes the same lower bound $\left(2^{n^{2} / 54-o\left(n^{2}\right)}\right)$ on the number of triangulations of $K_{n}$ for $n \equiv 9(\bmod 18)$.

Again the result follows immediately from these estimates and the Theorem.

Corollary 3.1.3 The constant $1 / 54$ that appears in the exponent in Corollary 3.1.2 may be improved to $2 / 81$ for $n \equiv 1,19,25$ or $27(\bmod 54)$

Proof. This follows by reapplying the $n \rightarrow 3 n-2$ and $n \rightarrow 3 n$ recursive constructions for triangulations as indicated in [2] and [7]. 


\section{Remarks.}

A new recursive construction for triangulations by two of the present authors, as yet unpublished, takes a face 2-colourable triangulation of $K_{n}$ and produces a face 2-colourable triangulation of $K_{3 n}$ without the need for the original triangulation to have a parallel class. This enables us to extend the result of Corollary 3.1 .2 to include $n \equiv 3(\bmod 18)$ and, by reapplication, to extend the result of Corollary 3.1.3 to include $n \equiv 3,7,9$ and $21(\bmod 54)$.

It is also possible to use some of the other constructions given in [7] to obtain lower bounds of the form $2^{a n^{2}-o\left(n^{2}\right)}$ for the number of Hamiltonian embeddings of $K_{n}$ for certain values of $n$ within the remaining residue classes.

\section{References}

[1] G. K. Bennett, Topological embeddings of Steiner triple systems and associated problems in design theory, Ph.D thesis, The Open University, 2004.

[2] C. P. Bonnington, M. J. Grannell, T. S. Griggs and J. Širáň, Exponential families of nonisomorphic triangulations of complete graphs, J. Combin. Theory Ser. B 78 (2000), 169-184.

[3] C. J. Colbourn and A. Rosa, Triple Systems, Clarendon Press, 1999.

[4] P. M. Ducrocq and F. Sterboul, On G-triple systems, Publications du Laboratoire de Calcul de l'Université des Sciences et Techniques de Lille, No. 103 (1978).

[5] M. N. Ellingham and C. Stephens, The nonorientable genus of joins of complete graphs with large edgeless graphs, submitted for publication.

[6] M. J. Grannell, T. S. Griggs and J. Širán̆, Surface embeddings of Steiner triple systems, J. Combin. Des. 6 (1998), 325-336.

[7] M. J. Grannell, T. S. Griggs and J. Šráň, Recursive constructions for triangulations, J. Graph Theory 39 (2002), no. 2, 87-107.

[8] M. J. Grannell and V. P. Korzhik, Nonorientable biembeddings of Steiner triple systems, Discrete Math. 285 (2004), 121-126.

[9] J. L. Gross and T. W. Tucker, "Topological Graph Theory", John Wiley, New York, 1987.

[10] V. P. Korzhik and H-J. Voss, Exponential families of nonisomorphic nonorientable genus embeddings of complete graphs, J. Combin. Theory Ser. B, 91 (2004), 253-287. 
[11] G. Ringel, "Map color theorem", Springer-Verlag, New York and Berlin, 1974.

[12] J. W. T. Youngs, The mystery of the Heawood conjecture, in: "Graph Theory and its Applications" (B. Harris, Ed.), Acad. Press, 1970, 17-50. 